\title{
Perioperative pain management for general and plastic surgery
}

\author{
Deepak Narayan, Alan D. Kaye, Nalini Vadivelu (Editors). Oxford University Press 2019. \\ New York. Price: \$78.39 (Hardcover); 288 pages. ISBN 978-0-19-045700-6
}

\author{
Claudia P. Gomez, MD iD
}

Received: 7 December 2018/Accepted: 8 December 2018/Published online: 18 January 2019

(C) Canadian Anesthesiologists' Society 2019

Perioperative pain management is a complex area in patient care. Any patient scheduled for a surgical procedure requires a preoperative pain assessment and treatment optimization plan. This plan should include educating the patient about expectations as well as addressing the various options for pain management. In addition, the impact of concurrent medications on pain management and knowledge of the patient's concomitant conditions (whether physiological, psychological, or both) could help clarify the specific strategy for pain control. Some such conditions include the presence of sleep apnea or factors linked to a predisposition for persistent postoperative pain, such as catastrophizing or anxiety.

The editors' purpose for this concise, evidence-based handbook was to provide both general and plastic surgeons with the essential information about effective, safe management of patients with pain. The editors, Dr. Deepak Narayan, a professor of surgery, and Drs. Alan D. Kaye and Nalini Vadivelu, both anesthesiologists, recruited experts in anesthesiology, pain medicine, plastic surgery, general surgery, and psychiatry to write the various chapters for these sections.

Perioperative Pain Management for General and Plastic Surgery is divided into 22 chapters with topics ranging from pain pathways and pain physiology to the surgical treatment of migraine headaches. Several chapters are dedicated to pharmacology, covering various types of analgesics used during the perioperative period, including non-steroidal anti-inflammatory drugs, Nmethyl-D-aspartate receptor antagonists, and long-acting opioids. Two chapters are dedicated to describing peripheral nerve blocks in both adult and pediatric populations. The book also addresses side effects and the

C. P. Gomez, MD ( $)$

Faculty of Medicine, University of Ottawa, Ottawa, ON, Canada consequences of inappropriate pain management, including barriers to optimal pain management. The book's illustrations and tables are all in black and white, which, although informative, lack some visual appeal.

Each section offers a succinct, accurate review of the given topic, including a complete reference list. In the case of the chapter, "Barriers to Optimal Pain Management in the General Surgery Population," the authors included a case study that could be used in the academic context to start a discussion with a learner. This is but one example of clinically important topics that touch on multimodal analgesia, management of neuropathic postoperative pain, and pain management in patients with substance abuse disorder. The authors offer specific recommendations for pain management in these common situations.

Given the various potential readers of the book, focusing on plastic and general surgeons, it is evident that this handbook is not meant to be read cover to cover. An example is the chapter entitled "Pain Management in Body Contouring Procedures," which describes a procedure almost exclusively in the domain of plastic surgeons.

There are several limitations that could be addressed in future editions. For example, it would have been useful if the content of the book had been differently organized perhaps according to specific specialties and topics. For example, chapters focusing on the pharmacology of analgesics and related adjuncts appear in several areas of the handbook, with some of the subjects overlapping and repeated throughout the book, such as two chapters addressing barriers to optimal pain management. Also, inclusion of a summary with recommendations for specific dosing of medications in the chapters focusing on pharmacology would help with the intended objective of providing essential information to surgeons.

In summary, Perioperative Pain Management for General and Plastic Surgery provides general and plastic surgeons with general guidelines for perioperative pain 
management in specific situations. It contributes to the general understanding of potential complications of inadequate pain management in various surgical populations.
Editorial responsibility This submission was handled by Dr. Hilary P. Grocott, Editor-in-Chief, Canadian Journal of Anesthesia.

Publisher's Note Springer Nature remains neutral with regard to jurisdictional claims in published maps and institutional affiliations.

Conflicts of interest None declared. 